should work to assist and help research at the institution, not be a hindrance.

Nancy A. Johnston* and Keely Szilágyi Indiana University School of Medicine, Indianapolis, Indiana, USA.

*e-mail: johnstna@iu.edu

Published online: 21 August 2019

https://doi.org/10.1038/s41684-019-0373-Z
References

. Institute for Laboratory Animal Research. Guide for the Care and Use of Laboratory Animals 8th Ed. P. 24. (National Academies Press, Washington, DC, 2011)

2. Silverman, J., Suckow, M.A., and Murthy, S. The IACUC Handbook 3rd Ed. P. 156, 9:34. (CRC Press, Boca Raton, FL, 2014)

3. Public Health Service. Policy on Humane Care and Use of Laboratory Animals. Section B.4. (US Department of Health and Human Services, Washington, DC, 1986, revised 2015).

4. Silverman, J., Suckow, M.A., and Murthy, S. The IACUC Handbook 3rd Ed. P. 281-282, 14:37, 14:38. (CRC Press, Boca Raton, FL, 2014)
5. Public Health Service. Policy on Humane Care and Use of Laboratory Animals. Section C.2. (US Department of Health and Human Services, Washington, DC, 1986, revised 2015).

6. Silverman, J., Suckow, M.A., and Murthy, S. The IACUC Handbook 3rd Ed. P. 95, 6:29. (CRC Press, Boca Raton, FL, 2014).

7. Silverman, J., Suckow, M.A., and Murthy, S. The IACUC Handbook 3rd Ed. P. 706, 29:45. (CRC Press, Boca Raton, FL, 2014).

8. Public Health Service. Policy on Humane Care and Use of Laboratory Animals. Section C.7. (US Department of Health and Human Services, Washington, DC, 1986, revised 2015).

9. Institute for Laboratory Animal Research. Guide for the Care and Use of Laboratory Animals 8th Ed. P. 16-17. (National Academies Press, Washington, DC, 2011)

\title{
Conflicts of interest: Lack of policy and/or of common sense?
}

W hile the rules governing animal research can always be applied to the proverbial "letter"

by IACUCs, some common sense and pragmatic dialogue is required to maintain a productive partnership between the regulators and scientists and ensure both compliance and research progress. This case is a disappointing application of the rules for addressing a member's or committee's personal agenda against Monteverde. In the absence of such conflicts of interest (COIs), this situation could have been resolved without fanfare or drama as Monteverde had every intent to comply with established procedures. This scenario highlights a need for guidance on eliminating detrimental COIs from interfering with a researchIACUC partnership that was intended to further the biomedical research enterprise.

According to the scenario, Monteverde was informed verbally by IACUC administration that the protocol was approved and that he could begin the work. It was later discovered that the IACUC erred in not sending a written letter within the next day, as the local IACUC's own policy states. Monteverde relied on the "word" of the committee and had no intention to circumvent any federal guidelines. A critical aspect is that animal safety was assured and not in jeopardy. The subsequent actions and sanctions from the IACUC are then quite problematic. The actions were initiated by an IACUC member, denoted IACUC member $Y$, who has had personal conflicts with Monteverde. At its worse, this is a manipulation of the rules structure and a recruitment of other committee members to satisfy IACUC member Y's personal agenda.
A simple self-recusal on all matters by IACUC member $Y$ would have removed the spectacle of tainting the proceedings with personal issues. Monteverde had all intention to comply with the protocol and IACUC rules. The personal conflict by an IACUC member created a more devastating situation that resulted in excessive punishment and sanction. Yes, the rules can always be applied in this way by the letter of the rules, but such use is also contrary to the spirit on which the rules were devised.

In the end, this is a case of miscommunication and of a personal agenda by IACUC member Y. The sanctions on Monteverde are overly harsh. The IACUC committee wields considerable power over an investigator's research. Some common sense judgment should be exercised in wielding those powers, which should never be subverted to satisfy a personal agenda against an individual investigator. A flaw is that the IACUC proceedings do not have sufficient language or guidance on the basis for recusals on a wide range of COIs. Thus, such overreach of possible powers and of sanctions are technically possible, but should they be used? This is akin to enacting a life imprisonment for a traffic violation. This overly harsh application then victimizes investigators such as Monteverde, who submitted the protocols and acted in good faith on the communications that he received. A good dose of ethical behavior, common sense, and adequate communication by the IACUC would have avoided this entire debacle.

Recommendation: Specific guidance on recusal of IACUC members is required to avoid a subversion of the process for personal and institutional agendas not related to the protocol at hand. While most guidance should be simple common sense, this scenario and the practice of levying disproportionately harsh sanctions are detrimental to the partnership of IACUCs and research programs. It is unconscionable that a simple miscommunication and bureaucratic snafu can lead to a suspension and interference with a PI's research. Judicious and common sense exercise of IACUC committee rules should be the objective of every committee. Not all powers should be wielded to the utmost degree. But, the gap in specific guidance on COI provides a loophole to subvert the process for personal agendas by a specific member. This member then swayed his fellow committee members-IACUC member Y should not have participated at all. Specific guidance on conflicts of interest and for member recusals is necessary. We must close this loophole with thoughtful new policies at the institutional and federal level. The IACUC and research programs should be a partnership to advance our biomedical research enterprise. The IACUC should never be used as a vehicle for personal agendas and should be exceptionally mindful of the spirit in which the rules were devised.

\section{Amy S. Yee}

Department of Developmental, Molecular and Chemical Biology, Tufts University School of Medicine, Boston, MA, USA.

e-mail:amy.yee@tufts.edu

Published online: 21 August 2019 https://doi.org/10.1038/s41684-019-0375-x 